\title{
Agrupamento de solos quanto à salinidade no Perímetro Irrigado de Custódia em função do tempo ${ }^{1}$
}

\author{
Maria B. G. S. Freire ${ }^{2}$, Márcio F. A. Miranda ${ }^{3}$, Emanoel E. M. Oliveira ${ }^{4}$, \\ Luís E. da Silva ${ }^{5}$, Luiz G. M. Pessoa ${ }^{6}$ \& Brivaldo G. de Almeida ${ }^{7}$ \\ ${ }^{1}$ Trabalho selecionado do II INOVAGRI International Meeting, realizado de 13 a 16 de abril de 2014, Fortaleza - CE, Brasil. Trabalho extraído da Tese do segundo autor \\ ${ }^{2}$ Departamento de Agronomia/UFRPE. Recife, PE. Bolsista PQ/CNPq. E-mail: betania@depa.ufrpe.br (Autor correspondente) \\ ${ }^{3}$ Departamento de Agronomia/UFRPE, Recife, PE. E-mail: marciofam@gmail.com \\ ${ }^{4}$ Departamento de Agronomia/UFRPE, Recife, PE. E-mail: ernandes.medeiros@hotmail.com \\ ${ }^{5}$ Departamento de Agronomia/UFRPE, Recife, PE. E-mail: luiseduardo.les87@yahoo.com.br \\ ${ }^{6}$ Pós-Doutorando PDJ-CAPES/CNPq Ciência Sem Fronteiras, Departamento de Agronomia, UFRPE, Recife, PE. E-mail: pessoa.lgm@gmail.com \\ ${ }^{7}$ Departamento de Agronomia/UFRPE, Recife, PE. E-mail: brivaldo@depa.ufrpe.br
}

\section{Palavras-chave:}

estatística multivariada

degradação

qualidade do solo

\section{R E S U M O}

Solos em perímetros irrigados sob clima semiárido são propensos ao acúmulo de sais e, no Brasil, este problema tem sido expressivo no semiárido do Nordeste, no entanto, poucos dados existem da real situação desta degradação. O objetivo deste trabalho foi avaliar a salinidade e sodicidade no Perímetro Irrigado de Custódia em diferentes épocas e determinar grupos de solos por meio de variáveis associadas à salinidade e sodicidade. Foram feitas amostragens de solos nos 55 lotes nas camadas de 0-10, 10-30 e 30-60 cm em quatro épocas, duas ao final do período chuvoso e duas ao término da época seca. Os resultados de $\mathrm{pH}$ em água, condutividade elétrica do extrato de saturação (CE), relação de adsorção de sódio (RAS) e percentagem de sódio trocável (PST) foram submetidos à estatística multivariada, por meio da técnica de agrupamento. Foram obtidos oito grupos de solos, que diferiram entre as épocas de amostragem e as variáveis avaliadas, predominando valores de $\mathrm{pH}$ na faixa alcalina, baixos valores de $\mathrm{CE}$, enquanto a RAS e a PST alternaram entre as épocas de amostragem, com valores que caracterizam a sodicidade. Os solos do Perímetro Irrigado de Custódia (PE) são alcalinos, de baixa salinidade e de média a alta sodicidade, susceptíveis à dispersão de coloides e degradação de sua qualidade física.

\section{Key words:}

multivariate statistics

degradation

soil quality

\section{Grouping of soils in relation to salinity in Irrigated Perimeter of Custódia in function of time}

\begin{abstract}
A B S T R A C T
Soils in irrigated perimeters under semiarid climate are inclined to accumulate of salts and in Brazil, this problem has been impressive in the semiarid Northeast, however, few data exist on the actual situation of this degradation. The aim of this study was to evaluate the salinity and sodicity in the Irrigated Perimeter of Custodia in different seasons and determine soil groups by variables associated with salinity and sodicity. Soil samples were taken in 55 plots at 0-10, 10-30 and 30-60 cm depth in four seasons, two at end of the rainy season and two at the end of the dry period. The results of $\mathrm{pH}$, electrical conductivity of the saturation extract (CE), sodium adsorption ratio (RAS) and exchangeable sodium percentage (PST) were subjected to multivariate statistics, through clustering technique. Eight groups of soils that differed between sampling times and the variables studied, predominantly in the alkaline $\mathrm{pH}$ range, low CE values were obtained, while the RAS and PST alternated between sampling times, with values that characterize sodicity. Soils of the Custódia Irrigated Perimeter (PE) are alkaline, of low salinity and medium to high sodicity, susceptible to dispersion of colloids and degradation of its physical quality.
\end{abstract}

\section{INTRODUÇÃo}

O acúmulo de sais em solos ocorre em função de fatores, como: condições climáticas onde os índices de evapotranspiração superam os de precipitação (típica em regiões de clima árido e semiárido), drenagem deficiente, riqueza do material de origem, etc. Áreas afetadas por sais ocorrem em todo o mundo, principalmente em regiões de clima árido e semiárido, onde a irrigação é fundamental para uma agricultura bem sucedida. Este avanço da irrigação nas regiões semiáridas em áreas de terras marginais e com o uso de águas de baixa qualidade tem incrementado o acúmulo de sais nos solos e, consequentemente, sua degradação (Ribeiro, 2010).

Elevadas concentrações de sais nos solos constituem um dos problemas para a agricultura global, com graves consequências econômicas e sociais (Farifteh et al., 2007). Em nível mundial, mais de 800 milhões de hectares de terras são estimados como 
sendo áreas afetadas por sais, correspondendo a mais de $6 \%$ dos solos aráveis (Muns \& Tester, 2008).

Este problema vem se intensificando especialmente em áreas irrigadas, devido ao manejo inadequado do sistema solo-água-planta, o que reflete na redução da produtividade dos cultivos. No Nordeste do Brasil, aproximadamente nove milhões de hectares de solos são afetados por sais (Fageria \& Gheyi, 1997). Nestas áreas, havendo uma enorme carência de água, foram construídos açudes com elevadas capacidades de armazenamento de água e nas proximidades construídos perímetros irrigados que, em sua maioria tem apresentado o problema da salinização dos solos. Segundo Gheyi (2000), na região Nordeste do Brasil aproximadamente 25\% das áreas irrigadas encontram-se salinizadas.

A escolha de áreas para compor perímetros irrigados nem sempre foi suficientemente embasada em critérios técnicoeconômicos (Oliveira et al., 2002). Ainda de acordo com estes autores, este fato, associado à falta de habilidade de parte dos irrigantes no manejo da irrigação e às peculiaridades edafoclimáticas do Nordeste brasileiro, muito têm contribuído para o processo de degradação de solos da região.

O Perímetro Irrigado de Custódia localiza-se no município de Custódia, Microrregião do Sertão do Moxotó, Pernambuco. Situado a $330 \mathrm{~km}$ da capital do estado (Recife), com coordenadas geográficas: $8^{\circ} 5^{\prime}$ 0" de latitude Sul e $37^{\circ} 37^{\prime} 30^{\prime \prime}$ de longitude Oeste e encontra-se a $540 \mathrm{~m}$ acima do nível do mar. Inserido em região de clima semiárido, com pluviosidade média anual de 570 $\mathrm{mm}$, a área do perímetro é relativamente plana, predominando relevo suave-ondulado. Os solos mais frequentes na área são os Neossolos Flúvicos, com ocorrência de Neossolos Litólicos e Vertissolos. Tem área irrigável correspondente a 350 ha, além de 991 ha de área de sequeiro, totalizando 1.341 ha, dividida em 55 lotes de pequenos produtores, com área média por lote de 4,3 ha. O principal sistema de irrigação utilizado no perímetro é por superfície em sulco (gravidade), na produção de banana, tomate industrial, milho industrial e feijão phaseolus (DNOCS, 2008).

Segundo Santos \& Araujo Filho (2008) a área do perímetro está classificada como terras não aráveis com problemas de drenagem (legenda $6 \mathrm{~d}$ ), solos altamente susceptíveis à salinização e sodificação, principalmente em regiões de clima árido e semiárido quando submetidos à irrigação com águas de teores elevados de sais (USSL Staff, 1954; Ayers \& Westcot, 1985). No entanto, a implantação do perímetro foi iniciada em 1974 e concluída em 1975, sendo a operação datada de 1975. Assim, o objetivo deste trabalho foi definir classes de solos afetados por sais no Perímetro Irrigado de Custódia, com base nas propriedades destes solos relacionadas à salinidade.

\section{MATERIAL E Métodos}

O trabalho foi desenvolvido no Perímetro Irrigado de Custódia, localizado no município de Custódia, PE. Na avaliação da qualidade dos solos da área, foram coletadas amostras em todos os 55 lotes que compõem o Perímetro. A amostragem adotada foi do tipo aleatória (Meunier et al., 2001), tomando-se uma amostra de solo por lote em ponto georreferenciado (Figura 1).

Foram realizadas quatro amostragens, em duas épocas do ano, sendo duas após o período chuvoso (julho/2009 e julho/2010) e duas ao final do período seco (janeiro/2010 e janeiro/2011), nas profundidades de 0-10, 10-30 e 30-60 cm, com amostras simples em um ponto de cada lote. Procurou-

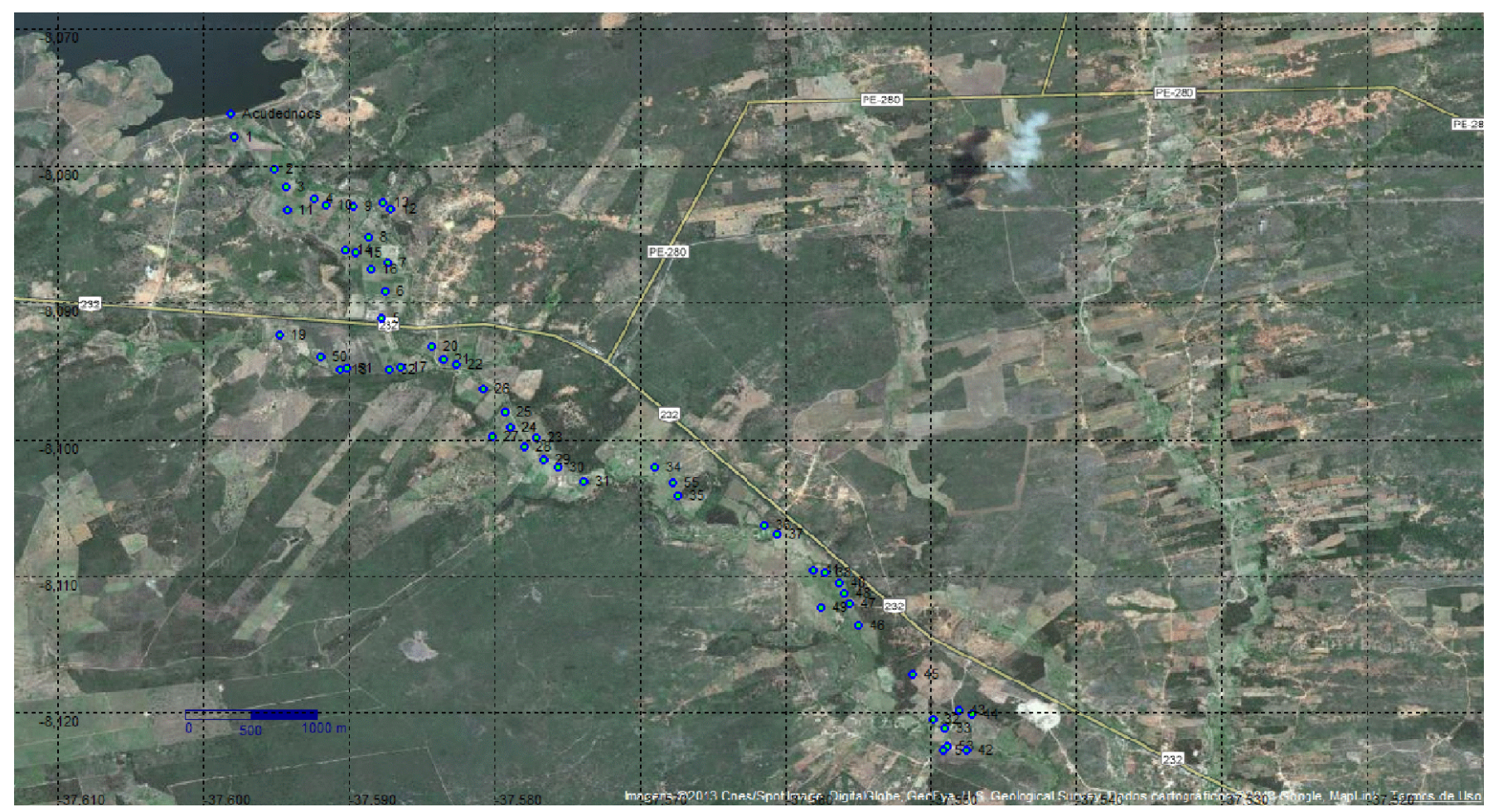

Figura 1. Imagem de satélite com a localização dos pontos de amostragem (em azul) no Perímetro Irrigado de Custódia, PE 


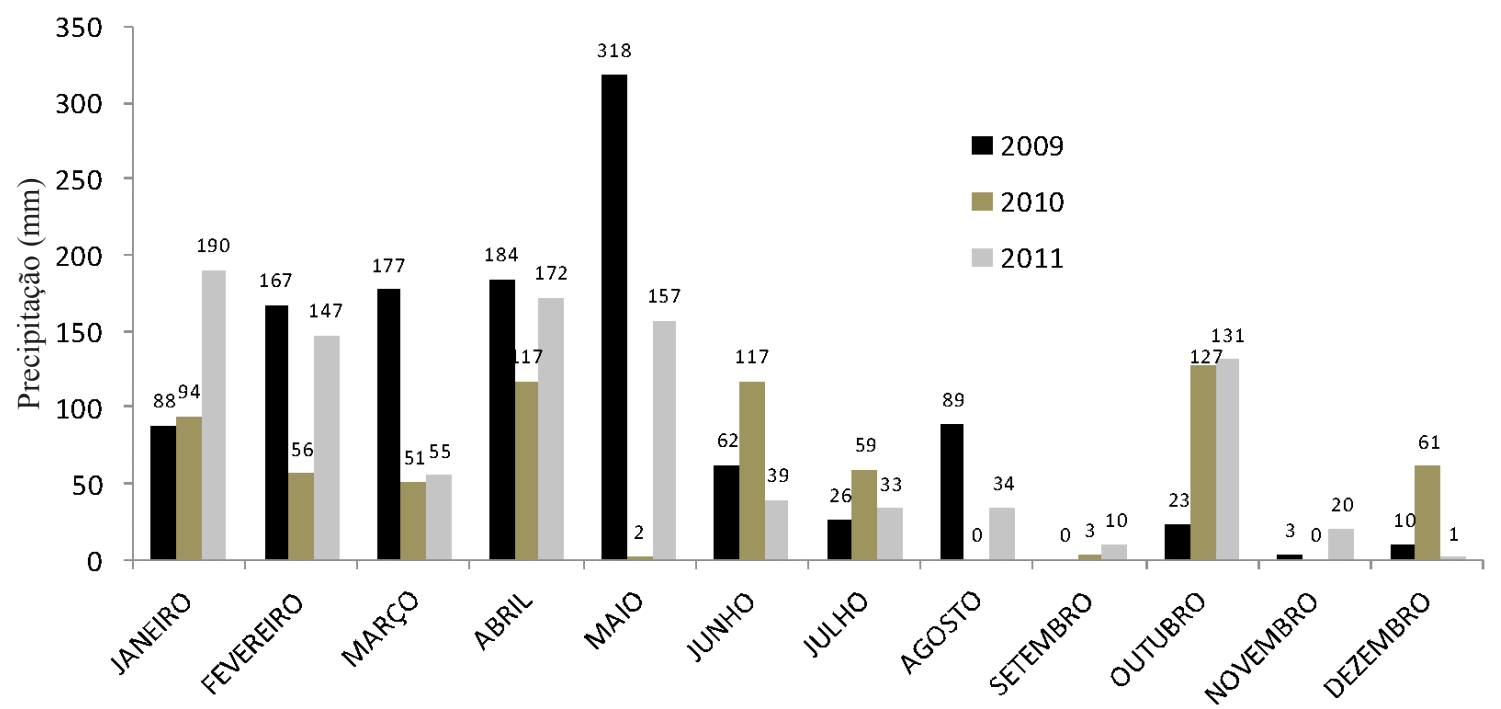

Figura 2. Médias de precipitação mensal registradas no município de Custódia, PE, durante a condução do trabalho (IPA, 2012)

se associar as amostragens dos solos com o regime hídrico da região, porque a movimentação de sais nos solos ocorre em função da passagem de água no perfil do solo. Os dados de precipitação mensal nos anos 2009, 2010 e 2011 encontram-se na Figura 2.

Para a avaliação dos atributos químicos relacionados à salinidade e sodicidade, procedeu-se o preparo da terra fina seca ao ar (TFSA), em que as amostras de solo foram secas ao ar, destorroadas e passadas em peneira com malhas de $2 \mathrm{~mm}$. Posteriormente, realizou-se a medida do $\mathrm{pH}$ em água $(1: 2,5)$, conforme EMBRAPA (1997).

$\mathrm{Na}$ avaliação dos sais solúveis, foram preparadas as pastas de saturação para a obtenção do extrato em sistema a vácuo. No extrato da pasta saturada, mediu-se a condutividade elétrica $\left(\mathrm{CE}\right.$ a $\left.25^{\circ} \mathrm{C}\right)$ e determinaram-se os teores dos cátions solúveis: $\mathrm{Na}^{+}$(fotometria de emissão de chama), $\mathrm{Ca}^{2+}{\mathrm{e} \mathrm{Mg}^{2+}}^{2+}$ (titulometria). Com os resultados destes cátions solúveis foi calculada a relação de adsorção de sódio (RAS) dos solos (USSL Staff, 1954).

Para o cálculo da percentagem de sódio trocável (PST), foi determinado o teor de $\mathrm{Na}^{+}$trocável, extraído com solução de acetato de amônio $1 \mathrm{~mol} \mathrm{~L}^{-1}$ e determinado por fotometria de emissão de chama; e a capacidade de troca de cátions, pelo método do acetato de sódio e acetato de amônio $1 \mathrm{~mol} \mathrm{~L}^{-1}$ (USSL Staff, 1954).

Foram utilizadas as variáveis $\mathrm{pH}$ do solo, CE, RAS e PST, pois são estas as indicadas para a definição de solos afetados por sais e sua classificação quanto à salinidade e sodicidade. Devido ao grande número de dados em três camadas distintas de solo, selecionou-se a última camada avaliada $(30-60 \mathrm{~cm})$ para a definição dos grupos de solos conforme as variáveis citadas, pois, na avaliação dos resultados das três camadas, a de $30-60 \mathrm{~cm}$ foi a que teve maiores valores de $\mathrm{pH}, \mathrm{CE}, \mathrm{PST}$ e RAS.

Os procedimentos estatísticos multivariados constaram da análise de agrupamento, a qual engloba uma variedade de técnicas e algoritmos, sendo que o objetivo é encontrar e separar objetos em grupos similares. A medida de dissimilaridade utilizada foi a distância euclidiana e o algoritmo de WARD como método de agrupamento.

O resultado da análise de agrupamento foi apresentado em gráfico na forma de árvore, conhecido como dendrograma. Este gráfico auxiliou na identificação dos agrupamentos dos lotes do perímetro mais semelhantes. A quantidade de grupos foi definida pela partição do dendrograma na primeira maior distância de ligação obtida, identificada no gráfico das distâncias de ligação nos sucessivos passos da análise de agrupamento.

\section{Resultados E Discussão}

Os resultados das análises de agrupamento referentes aos 55 lotes do Perímetro Irrigado de Custódia na profundidade de 30-60 cm em função da salinidade e sodicidade ( $\mathrm{pH}, \mathrm{CE}$, RAS e PST) dos solos nas quatro coletas realizadas estão representados na Figura 3. Devido as médias do pH, CE, RAS e PST serem mais elevadas na camada de $30-60 \mathrm{~cm}$, optou-se por realizar a análise de agrupamento apenas com os dados desta camada.

Nos dendrogramas da Figura 3, a escala vertical indica o nível de similaridade e no eixo horizontal são marcadas as amostras dos lotes agrupadas em função das variáveis utilizadas na caracterização dos sais dos solos (pH, CE, RAS e PST). Estas variáveis, em geral, não são independentes, atuam conjuntamente na definição da degradação dos solos e, por isso, foram analisadas conjuntamente.

Esta análise de agrupamento foi realizada para verificar a formação dos grupos no dendrograma, ou seja, aquelas amostras que possuíam valores médios aproximados de $\mathrm{pH}$, CE, RAS e PST, as quais estão relacionadas com a salinidade e sodicidade do solo, formaram grupos homogêneos; enquanto que as variáveis que possuíam uma média diferenciada das demais, formaram grupos heterogêneos.

Nos dendrogramas, os grupos foram definidos pelo traçado de uma linha paralela ao eixo horizontal, a qual foi definida com 
A.

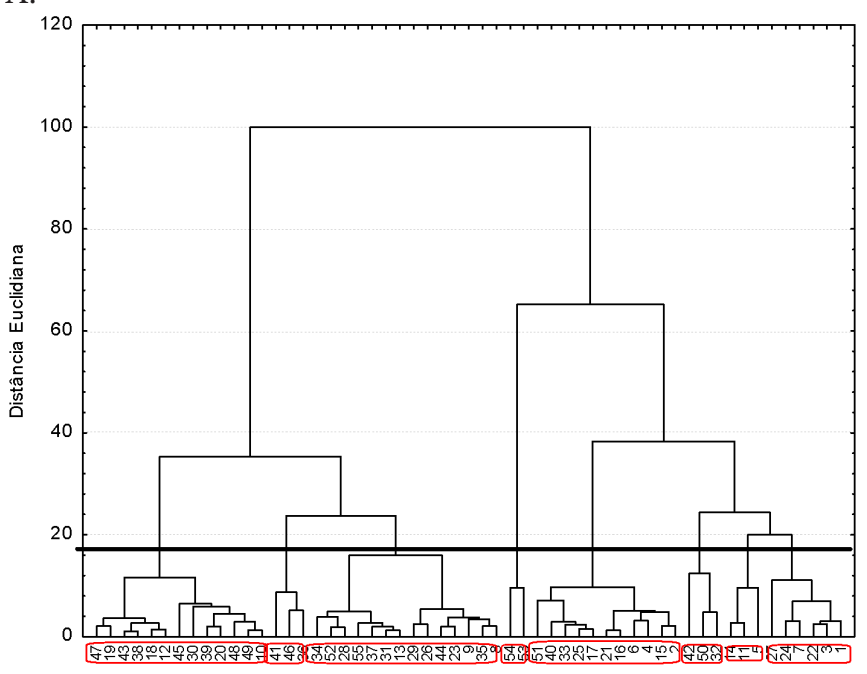

C.

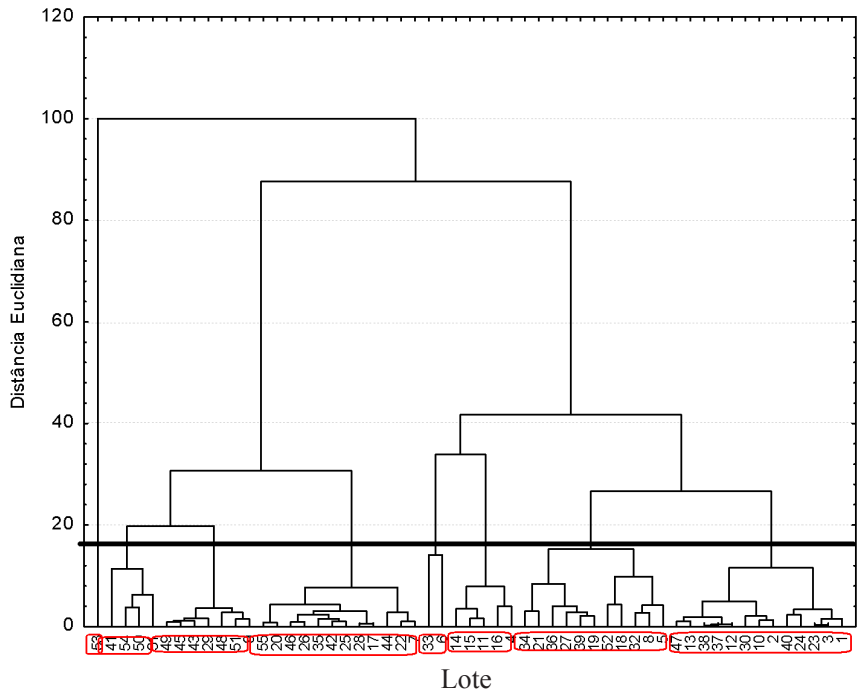

B.

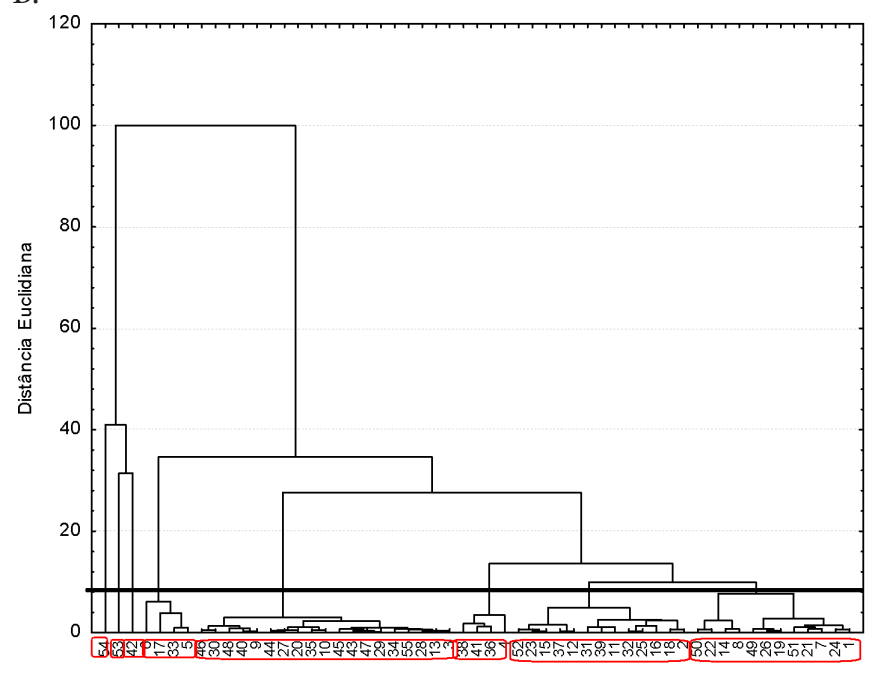

D.

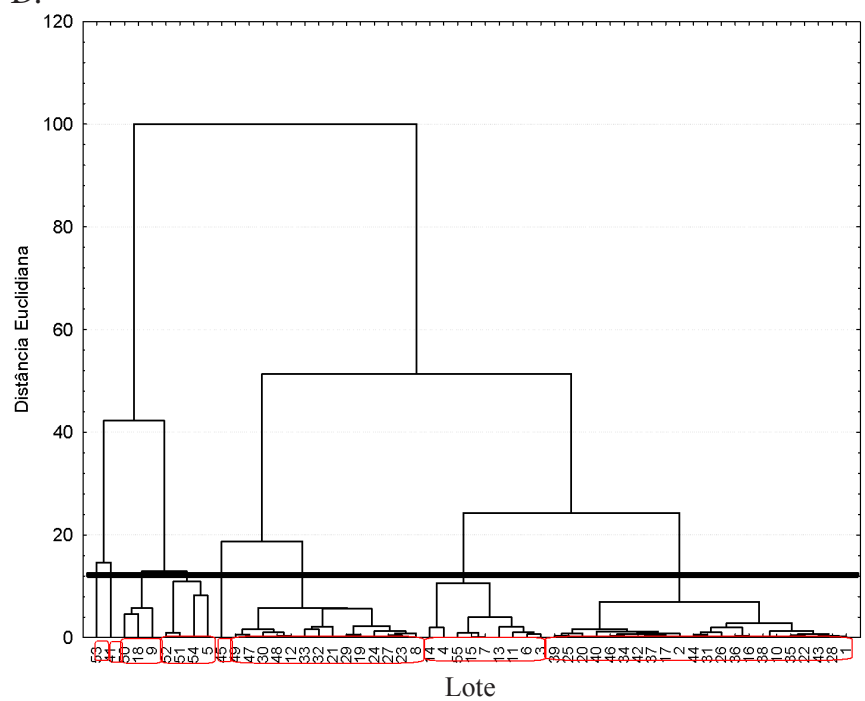

Figura 3. Dendrograma resultante da análise de agrupamento dos valores de $\mathrm{pH}, \mathrm{CE}, \mathrm{RAS}$ e PST dos 55 lotes do Perímetro Irrigado de Custódia, (A) coleta de julho de 2009, (B) coleta de janeiro de 2010, (C) coleta de julho de 2010 e (D) coleta de janeiro de 2011, na profundidade de 30-60 cm, utilizando a distância euclidiana como coeficiente de similaridade e o algoritmo de WARD como método de agrupamento

base no critério da maior distância entre os grupos (primeiro maior pulo), sendo definida a formação de oito grupos nas quatro coletas realizadas, sendo a numeração dos grupos feita da direita para esquerda.

Na Figura 3A, o grupo 1, formado pelos lotes 1, 3, 22, 7, 24 e 27, são os lotes que possuem a maior semelhança no dendrograma, por possuírem a menor distância euclidiana. Em seguida, vem o grupo 2, representado pelos lotes 5, 11 e 14 e, assim, sucessivamente, sendo estas amostras agrupadas por ordem decrescente de semelhança, ou seja, o grupo 8 formou o último grupo do dendrograma, o qual manteve-se diferente dos demais grupos formados, pelo fato de possuir pouca semelhança em relação aos demais.

Levando-se em consideração os grupos formados na Figura 3, observa-se que os grupos são distintos em cada época de amostragem, ou seja, existe homogeneidade dentro de cada grupo e heterogeneidade entre os grupos, isto é, estão agrupados por características em comum.
Os lotes do perímetro irrigado onde ocorreram os maiores problemas de salinidade e sodicidade na primeira coleta foram os que compõem o grupo 5 (Tabela 1), os quais são formados pelos lotes 53 e 54, com valores de pH, CE, RAS e PST de 10,27, $8,66 \mathrm{dS} \mathrm{m}^{-1}, 85,07\left(\mathrm{mmol} \mathrm{L}^{-1}\right)^{0,5}$ e $63,82 \%$, respectivamente. Estes lotes do perímetro irrigado encontram-se em pousio, devido à degradação associada aos problemas de salinidade e sodicidade dos mesmos. Podem ser considerados degradados, pois as quatro variáveis os caracterizam como tal, desde o $\mathrm{pH}$ elevado, fora da faixa indicada para a maioria das culturas, a CE superior ao limite de salinidade, e a RAS e PST, indicando sua sodicidade.

Baseando-se na classificação do USSL Staff (1954), observase que o grupo 2 (lotes 5,11 e 14), o grupo 3 (lotes 32,50 e 42) e o grupo 7 (lotes 36,46 e 41), na primeira coleta, é formado por solos classificados como sódicos, o que corresponde a $16 \%$ dos lotes do perímetro irrigado (Tabela 1). Nos solos sódicos, a infiltração da água é reduzida e o controle da salinidade é um problema (Queiroz et al., 2010). 
Tabela 1. Valores médios de $\mathrm{pH}$, condutividade elétrica do extrato de saturação (CE), relação de adsorção de sódio (RAS) e percentagem de sódio trocável (PST) dos grupos formados na análise de agrupamento na profundidade de $30-60 \mathrm{~cm}$ nas quatro coletas realizadas

\begin{tabular}{|c|c|c|c|c|c|c|c|c|}
\hline \multirow{2}{*}{ Atributos } & \multicolumn{8}{|c|}{ Grupos formados na análise de agrupamento } \\
\hline & 1 & 2 & 3 & 4 & 5 & 6 & 7 & 8 \\
\hline & \multicolumn{8}{|c|}{ Coleta julho de 2009} \\
\hline $\mathrm{pH}^{1}$ & 7,96 & 8,95 & 8,22 & 8,10 & 10,27 & 7,57 & 7,71 & 7,37 \\
\hline $\mathrm{CE}^{2}\left(\mathrm{dSm}^{-1}\right)$ & 1,80 & 1,16 & 2,15 & 1,67 & 8,66 & 0,53 & 1,67 & 0,98 \\
\hline $\operatorname{RAS}^{3}\left(\mathrm{mmol} \mathrm{L}^{-1}\right)^{0,5}$ & 5,12 & 2,93 & 13,92 & 5,81 & 85,07 & 1,97 & 7,59 & 2,44 \\
\hline \multirow[t]{2}{*}{$\mathrm{PST}^{4}(\%)$} & 10,62 & 16,18 & 29,95 & 12,09 & 63,82 & 7,96 & 28,87 & 9,88 \\
\hline & \multicolumn{8}{|c|}{ Coleta janeiro de 2010} \\
\hline $\mathrm{pH}$ & 7,87 & 7,83 & 8,29 & 7,53 & 8,02 & 9,55 & 10,33 & 9,69 \\
\hline $\mathrm{CE}\left(\mathrm{dS} \mathrm{m} \mathrm{m}^{-1}\right)$ & 1,29 & 1,02 & 2,96 & 0,55 & 1,03 & 8,55 & 3,00 & 3,50 \\
\hline $\operatorname{RAS}\left(\mathrm{mmol} \mathrm{L}^{-1}\right)^{0,5}$ & 5,38 & 3,91 & 19,39 & 3,17 & 4,88 & 61,04 & 15,04 & 56,83 \\
\hline \multirow[t]{2}{*}{ PST (\%) } & 13,03 & 4,97 & 16,16 & 3,78 & 2,63 & 45,49 & 43,66 & 84,61 \\
\hline & \multicolumn{8}{|c|}{ Coleta julho de 2010} \\
\hline $\mathrm{pH}$ & 7,83 & 7,83 & 7,88 & 7,55 & 7,78 & 6,99 & 7,48 & 10,30 \\
\hline $\mathrm{CE}\left(\mathrm{dS} \mathrm{m}^{-1}\right)$ & 0,60 & 1,42 & 2,29 & 1,35 & 1,78 & 1,90 & 3,18 & 10,25 \\
\hline $\operatorname{RAS}\left(\mathrm{mmol}^{-1}\right)^{0,5}$ & 3,23 & 6,35 & 5,37 & 3,79 & 6,65 & 6,16 & 9,63 & 56,20 \\
\hline \multirow[t]{2}{*}{ PST (\%) } & 4,08 & 9,80 & 6,42 & 5,29 & 6,00 & 9,31 & 29,48 & 53,00 \\
\hline & \multicolumn{8}{|c|}{ Coleta janeiro de 2011} \\
\hline $\mathrm{pH}$ & 8,08 & 8,68 & 7,49 & 5,50 & 7,48 & 8,07 & 9,40 & 9,20 \\
\hline $\mathrm{CE}\left(\mathrm{dS} \mathrm{m} \mathrm{m}^{-1}\right)$ & 0,53 & 0,93 & 1,01 & 2,30 & 6,45 & 4,46 & 8,96 & 15,73 \\
\hline $\operatorname{RAS}\left(\mathrm{mmol} \mathrm{L}^{-1}\right)^{0,5}$ & 2,44 & 4,33 & 2,43 & 3,86 & 18,33 & 9,49 & 44,26 & 86,57 \\
\hline PST (\%) & 2,96 & 5,52 & 3,74 & 4,74 & 36,03 & 18,44 & 66,80 & 45,33 \\
\hline
\end{tabular}

1pH em água (1:2,5); ' $C$ Condutividade elétrica do extrato de saturação; ${ }^{3}$ Relação de adsorção de sódio; 4Percentagem de sódio trocável

Apenas os solos do grupo 5 foram classificados como salinosódicos, sendo composto por dois lotes na primeira coleta (Julho 2009), o 53 e o 54, pois a CE só superou o limite de salinidade nos solos destes lotes (Tabela 1, Figura 3A).

Contudo, verificou-se que os maiores problemas neste perímetro irrigado estão relacionados com os altos teores de $\mathrm{Na}^{+}$trocável, que refletem na PST dos solos. Para a primeira coleta de solos (Julho 2009), foram encontrados solos com PST superior a 15\%, nos lotes dos grupos 2, 3, 5 e 7 , correspondendo a $20 \%$ dos lotes de todo o perímetro. O limite preconizado pelo USSL Staff (1954) como o que define solos sódicos é de $15 \%$ de PST, limite este também adotado pelo Sistema Brasileiro de Classificação de Solos (EMBRAPA, 2013). No entanto, Freire et al. (2003a) observaram que valores de PST inferiores a $15 \%$ já proporcionam degradação de solos similares aos deste Perímetro em Pernambuco. Se avaliarmos os solos com PST superior a 10\% nesta coleta (Tabela 1), mais de 50\% dos lotes estão acima deste valor de PST, o que torna preocupante o uso agrícola destas áreas.

Diversos estudos tem indicado o efeito do $\mathrm{Na}^{+}$na alteração da estrutura do solo com níveis inferiores aos 15\% de PST para solos sódicos, inclusive, para Pizarro (1985) é mais coerente considerar como sódicos solos com PST $>7 \%$. Pela classificação de Pizarro (1985), todos os oito grupos de solos deste perímetro irrigado na coleta de Julho de 2009 estariam com problemas de sodicidade, indo de ligeiramente sódicos (PST entre 7-10\%) até excessivamente sódicos (PST > 30\%).

Contudo, as respostas de cada solo aos teores de $\mathrm{Na}^{+}$ dependem de sua constituição, especialmente quanto aos teores de matéria orgânica, teores de argila e tipos de minerais presentes na fração argila (Freire et al., 2003a; b).

Nas demais coletas de solo (Janeiro 2010, Julho 2010 e Janeiro 2011), alguns resultados dos grupos foram alterados, em função de modificações provenientes de regime hídrico da região de localização do Perímetro (Figura 2, 3A e B). Entretanto, as observações foram praticamente as mesmas, em que a sodicidade foi mais intensa do que a salinidade (Tabela 1).

Nas amostras de solo de todas as coletas realizadas os sais de $\mathrm{Na}^{+}$estão presentes em proporções consideráveis em quase todos os lotes, tornando-os mais propensos a problemas na estrutura, como diminuição da permeabilidade ao ar e à água, que interferem na produção das culturas.

Resultados semelhantes aos encontrados nesta pesquisa também foram verificados por Fernandes (2008), no Perímetro Irrigado Cachoeira II, Serra Talhada, PE; e por Santos (2010), no Perímetro Irrigado Icó Mandantes, Petrolândia - PE. No entanto, Pessoa (2012) por meio de amostragem aleatória em diferentes solos no semiárido de Pernambuco verificou valores de CE, RAS e PST muito superiores aos encontrados neste estudo. Isto indica o alto grau de degradação dos solos do semiárido pernambucano pela salinidade e sodicidade. Sendo assim, a separação destes solos em grupos contendo solos similares quimicamente pode contribuir para estratégias de manejo a serem adotadas para cada grupo de solo formado.

\section{Conclusões}

1. Os lotes do Perímetro Irrigado de Custódia - PE têm solos em diferentes estágios de degradação pelo acúmulo de sais.

2. Entre os cátions avaliados, o $\mathrm{Na}^{+}$foi o que esteve nas concentrações mais elevadas, principalmente nas camadas mais profundas do solo.

3. O Perímetro Irrigado de Custódia tem solos com valores de RAS e PST que caracterizam solos sódicos e aumentam em profundidade em todas as coletas realizadas, indicando 
que os problemas neste Perímetro estão mais relacionados à sodicidade do que à salinidade.

\section{Literatura Citada}

Ayers, R. S.; Westcot, D. W. Water quality for agriculture. Rome: FAO, 1985.174p.

DNOCS - Departamento Nacional de Obras contra as Secas. http://www.dnocs.gov.br. 29 Out. 2008.

EMBRAPA - Empresa Brasileira de Pesquisa Agropecuária. Manual de métodos de análise de solo. Rio de Janeiro: EMBRAPA, 1997. 212p.

EMBRAPA - Empresa Brasileira de Pesquisa Agropecuária. Sistema Brasileiro de Classificação de Solos. Brasília: EMBRAPA, 2013. 353p.

Fageria, N. K.; Gheyi, H. R. Efeitos dos sais sobre as plantas. In: Gheyi, H. R.; Queiroz, J. E.; Medeiros, J. F. de. (ed.). Manejo e controle da salinidade na agricultura irrigada. Campina Grande: UFPB/SBEA, 1997. p.125-131.

Farifteh, J.; van der Meer, F; Atzberger, C.; Carranza, E. J. M. Quantitative analysis of salt-affected soil reflectance spectra: A comparison of two adaptive methods (PLSR and ANN). Remote Sensing of Environment, v.110, p.59-78, 2007.

Fernandes, J. G. Características de águas e solos do Perímetro Irrigado Cachoeira II, Serra Talhada/PE. Recife: UFRPE. 2008. 77p. Dissertação Mestrado

Freire, M. B. G. dos S.; Ruiz, H. A.; Ribeiro, M. R.; Ferreira, P. A.; Alvarez V., V. H.; Freire, F, J. Condutividade hidráulica de solos de Pernambuco em resposta à condutividade elétrica e RAS da água de irrigação. Revista Brasileira de Engenharia Agrícola e Ambiental, v.7, p.45- 52, 2003a.

Freire, M. B. G. dos S.; Ruiz, H. A.; Ribeiro, M. R.; Ferreira, P. A.; Alvarez V., V. H.; Freire, F, J. Estimativa do risco de sodificação de solos de Pernambuco pelo uso de águas salinas de irrigação. Revista Brasileira de Engenharia Agrícola e Ambiental, v.7, p.227-232, 2003b.

Gheyi, H. R. Problemas de salinidade na agricultura irrigada. In: Oliveira, T. S. de; Assis Jr., R. N.; Romero, R. E.; Silva, J. R. C. (org). Agricultura, sustentabilidade e o semiárido. Viçosa: Folha de Viçosa/SBCS. v.1. 2000. p.329-346.
IPA - Instituto Agronômico de Pernambuco. Índices pluviomé-tricos. <http://www.ipa.br/indice_pluv. php\#calendario_indices $>$. 10 Jan. 2012.

Meunier, I. M. J.; Silva, J. A. A.; Ferreira, R. L. C. Inventário florestal: programas de estudo. Recife: UFRPE, 2001. $189 \mathrm{p}$.

Munns, R.; Tester, M. Mechanisms of salinity tolerance. Annual Review of Plant Biology, v.59, p.651-681, 2008.

Oliveira, L. B. de; Ribeiro, M. R.; Ferreira, M. da G. de V. X.; Lima, J. F. W. F. de; Marques, F. A. Interferências pedológicas aplicadas ao perímetro irrigado de Custódia, PE. Pesquisa Agropecuária Brasileira, v.37, p.1477-1486, 2002.

Pessoa, L. G. M. Analysis of salt-affected soil in semiarid landscapes of Pernambuco, Brazil. Recife: UFRPE. 2012. 124p. Tese Doutorado

Pizarro, F. Drenaje agrícola y recuperación de suelos salinos. Madrid: Ediciones Mundi-Prensa, 1985. 542p.

Queiroz, J. E.; Gonçalves, A. C. A.; Souto, J. S.; Folegatti, M. V. Avaliação e monitoramento da salinidade do solo. In: Gheyi, H. R.; Dias, N. da S.; Lacerda, C. F. de (ed.) Manejo da salinidade na agricultura: Estudos básicos e aplicados. Fortaleza: INCTSal, 2010. p.63-81.

Ribeiro, M. R. Origem e classificação dos solos afetados por sais. In: Gheyi, H. R.; Dias, N. da S.; Lacerda, C. F. de (ed). Manejo da salinidade na agricultura: Estudos básicos e aplicados. Fortaleza, INCTSal, 2010. p.11-19.

Santos, J. C. P. dos; Araujo Filho, J. C. de (ed.). Avaliação detalhada do potencial de terras para irrigação nas áreas de reassentamento de colonos do Projeto Jusante - área 3, Glória, BA. Recife: Embrapa Solos - UEP/NE, 2008. $112 \mathrm{p}$.

Santos, P. R. Atributos do solo em função dos diferentes usos adotados em Perímetro Irrigado do Sertão de Pernambuco. Recife: UFRPE. 2010. 113 p. Tese Doutorado

USSL - United States Salinity Laboratory Staff. Diagnosis and improvement of saline and alkali soils. Washington: US Department of Agriculture, 1954. 160p. USDA Agricultural Handbook, 60 